\title{
Malignant Transformation of a Multi-Operated Divided Nevus of the Eyelids
}

\author{
Stéphanie Lemaître ${ }^{a, b, e}$ Sophie Gardrat ${ }^{a, c}$ Anne Vincent-Salomon ${ }^{a, c}$ \\ Olivier Galatoire $^{d} \quad$ Christine Lévy-Gabriel $^{\mathrm{a}, \mathrm{e}} \quad$ Laurence Desjardins $^{\mathrm{a}}$ e \\ a Institut Curie, b Université Paris Descartes, cPathology and Tumor Biology, Institut Curie,

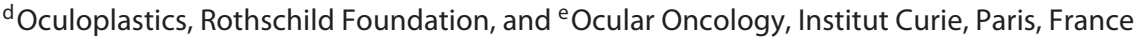

\section{Established Facts}

- Divided nevi of the eyelids are rare. Malignant transformation into melanoma is exceptional since only 2 cases have been reported so far.

\section{Novel Insights}

- This is the second case in the literature with a histological proof of the melanoma and the only case for which a genetic analysis was performed, showing an NRAS activating mutation.

\section{Keywords}

Divided nevus · Kissing nevus · Eyelid · Melanoma

\section{Abstract}

A woman in her early fifties had regular follow-up for a medium-sized divided nevus of the eyelids which had undergone several surgical excisions during childhood for functional and esthetic reasons. Malignant transformation of the nevus occurred in the inferior eyelid, with the appearance of a new pigmented flat lesion. Histology showed in situ melanoma, and an NRAS activating mutation was found. A full-thickness excision of the inferior eyelid was performed, followed by reconstruction. Local recurrence of the melanoma occurred 1 year after surgery. Lifelong fol-
๑) 2017 S. Karger AG, Basel

E-Mail karger@karger.com www.karger.com/oop low-up of divided nevi of the eyelids is recommended, even if very few cases of malignant transformation have been reported so far.

(c) 2017 S. Karger AG, Basel

\section{Introduction}

Divided nevi of the eyelids are rare congenital lesions. We report an exceptional case of malignant transformation into melanoma which occurred in adulthood.

This case was presented at the biennial Conference of the ISOO (International Society of Ocular Oncology), March 24-28, 2017, Sydney, Australia. 

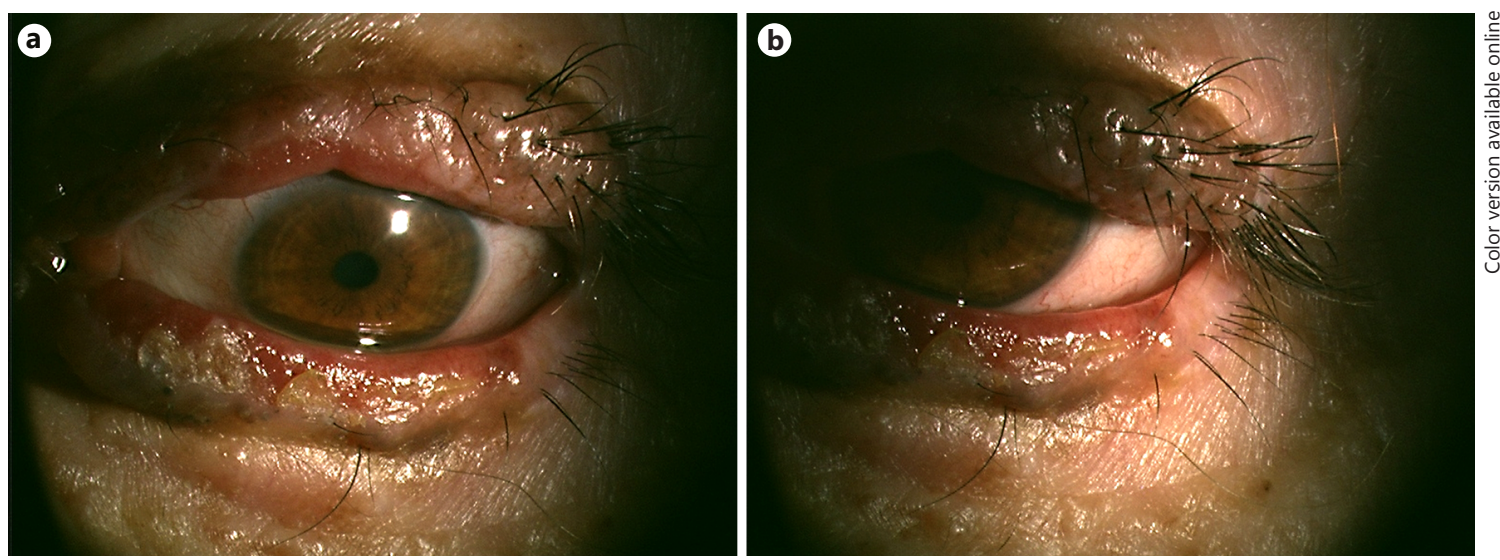

Fig. 1. a, b Residual nevus with cicatricial ectropion of the inferior eyelid due to previous surgeries. Prior to the excision surgeries, the initial lesion was a medium-sized divided nevus of the eyelids.

\section{Case Report}

A 53-year-old woman was referred to ocular oncology because of a persistent divided nevus of the left upper and lower eyelids with a history of multiple surgeries between the ages of 9 and 15 for cosmetic reasons. The initial lesion in childhood was a medium-sized congenital melanocytic nevus $[1,2]$. On examination, residual nevic tissue was present on both the upper and lower eyelids (Fig. 1a, b). Regular follow-up every 6 months was decided.

Four years later, the patient presented with a new pigmented lesion of the left inferior eyelid which had been evolving for 2 months (Fig. 2a). A biopsy of the lesion was performed and histological analysis showed in situ and invasive cutaneous melanoma (Fig. 2b-d). A full-thickness excision of the whole inferior eyelid allowed tumor clearance with 5- to 6-mm security margins according to the pathology report. Final histological diagnosis was superficial spreading melanoma (Clark level IV and Breslow thickness $1.9 \mathrm{~mm}$, stage T1aN0M01 [3]). An activating mutation was found on the NRAS gene (Gln61Arg), and no mutations were found on the BRAF (exons 11 and 15) and KIT genes (exons 11, 13, and 17). Lower eyelid reconstruction consisted of a superiorly based tarsoconjunctival advancement flap for posterior lamellar repair and a skin graft from the supraclavicular region for anterior lamellar repair.

The esthetic and functional aspects of the reconstructed inferior eyelid were satisfying after the surgery. There were no lagophthalmos or epiphora and a minimal scleral show was noted postoperatively (Fig. 2e). Local recurrence of in situ melanoma occurred 1 year after the surgery (Fig. $2 \mathrm{f}-\mathrm{g}$ ) requiring additional surgical excision. A spindle-shaped resection was performed and clear 3-mm margins were obtained.

\section{Discussion}

Divided nevi of the eyelids (also called "kissing nevi," "panda nevi," or "split ocular nevi") are rare congenital lesions that affect both the upper and lower lid and extend to the lid margin. These nevi are divided by the palpebral fissure. They form during embryological life as a single lesion when the eyelids are still fused, after the migration of melanoblasts from the neural crest to the epidermis (between the 12th and 14th week of gestation). The lids separate around the 24th week of gestation and the nevus is then divided as well [4].

BRAF and NRAS mutations are the most common mutations in nevi. These mutations occur in utero and therefore are not UV induced. They are almost always mutually exclusive, but mutation heterogeneity has been reported [5]. NRAS mutation is the sole recurrent somatic mutation in large and giant congenital melanocytic nevi, whereas small to medium lesions harbor a BRAF mutation in $30 \%$ of the cases [6]. NRAS mutations may allow a greater expansion of melanocytes than BRAF via an inhibition of apoptosis, which would account for its association with larger lesions [7].

The projected adult size of divided nevi of the eyelids rarely exceeds $10 \mathrm{~cm}[8,9]$, making them small to medium lesions according to the Ruiz-Maldonaldo classification. Large lesions are rare [10].

Malignant transformation of divided nevi into melanoma is exceptional. Only congenital melanocytic nevi larger than $20 \mathrm{~cm}$ in diameter (called "giant nevi") have a particularly high malignant risk; divided nevi are generally small to medium-sized and are thus at low risk [11]. To our knowledge, 2 cases of melanoma arising on a preexisting divided nevus have been reported to date. The first case was reported by Fuchs in 1950: the patient presented with a recurrent enlarging pigmented lesion which had occurred 6 years after excision surgery of a divided nevus, but no histological analysis was performed [12]. 

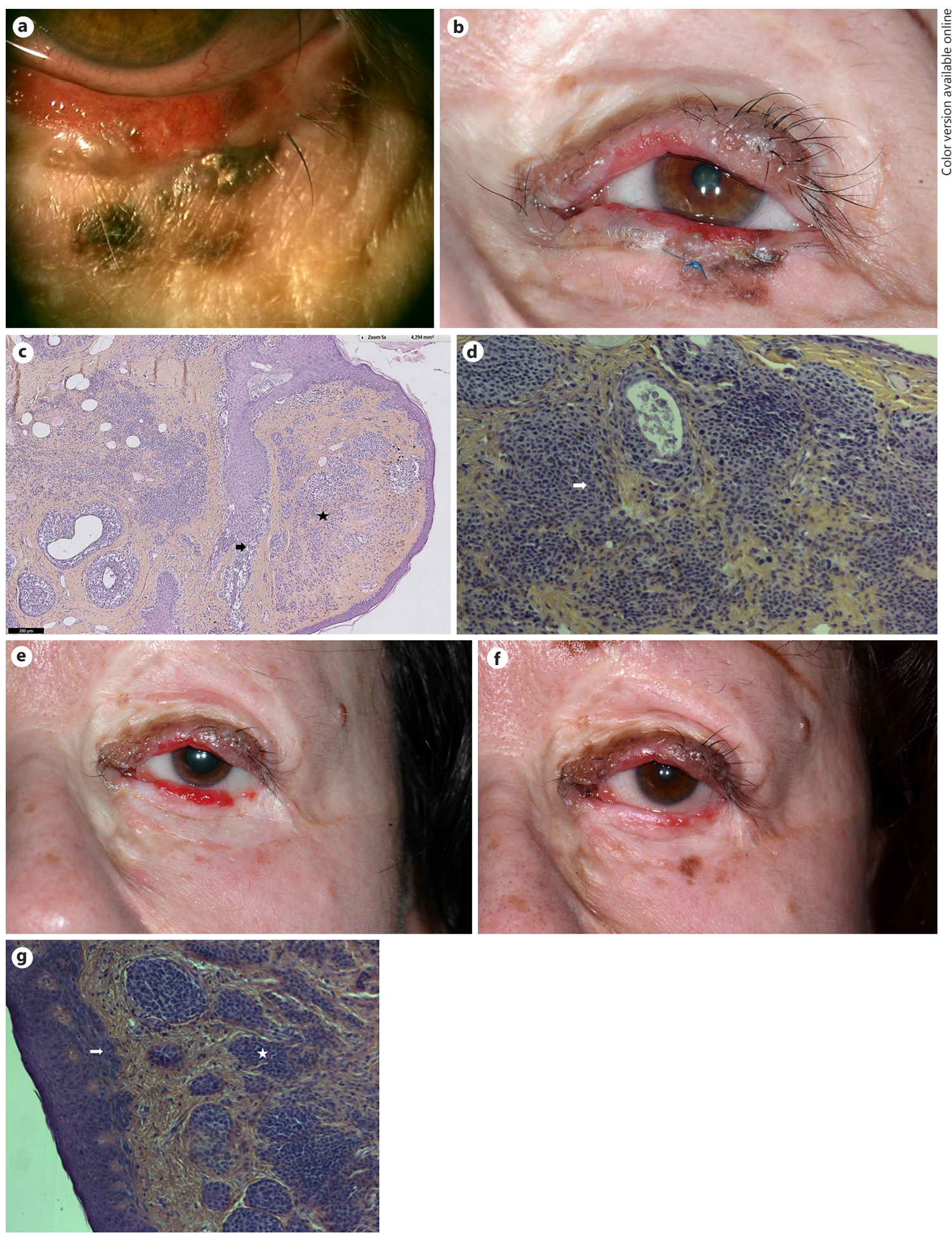

Fig. 2. a Heterogeneously pigmented skin lesion on the lower edge of the divided nevus (which is not visible in Fig. 1). b Same aspect as in a (photograph taken after the local biopsy of the suspicious lesion on the lower eyelid). c, $\mathbf{d}$ Histological section of the primary lesion showing in situ melanoma (black arrow), the underlying nevus (black star), and invasive melanoma (white arrow). e Aspect after complete excision of the melanoma and reconstruction of the inferior eyelid. $\mathbf{f}$ Local recurrence at the inferior part of the reconstructed eyelid. g Histological analysis showing in situ cutaneous melanoma (white arrow). There is an intraepidermal melanocytic proliferation of spindle cells with moderate cytonuclear atypia. The white star shows the associated nevus. 
The other case was reported in 2012: the transformation of a divided nevus into melanoma was confirmed by histology, and synchronous regional lymph node metastases were present [13].

Surgical debulking of divided nevi is performed mainly because of cosmetic or functional issues and in most cases, the lesion does not have to be entirely removed. Due to the low risk of malignant transformation, these partial excisions are acceptable [14]. Lifelong clinical follow-up is recommended.

\section{Statement of Ethics}

This report has been done according to the Declaration of Helsinki.

\section{Disclosure Statement}

The authors declare that there are no conflicts of interest.

\section{References}

1 Krengel S, Scope A, Dusza SW, Vonthein R, Marghoob AA: New recommendations for the categorization of cutaneous features of congenital melanocytic nevi. J Am Acad Dermatol 2013;68:441-451.

2 Ruiz-Maldonado R: Measuring congenital melanocytic nevi. Pediatr Dermatol 2004;21: 178-179.

3 Edge S, Byrd DR, Compton CC, Fritz AG, Greene FL, Trotti A (eds): AJCC Cancer Staging Manual. 7th edition. London/New York, Springer, 2011.

4 Rajput GC, Mahajan D, Chaudhary KP, Deewana V: Kissing naevus arising from neural crest cells presenting as upper and the lower lid mass. J Neurosci Rural Pract 2015;6:417419 .
5 Pollock PM, Harper UL, Hansen KS, Yudt LM, Stark M, Robbins CM, et al: High frequency of BRAF mutations in nevi. Nat Genet $2003 ; 33: 19-20$.

6 Charbel C, Fontaine RH, Malouf GG, Picard A, Kadlub N, El-Murr N, et al: NRAS mutation is the sole recurrent somatic mutation in large congenital melanocytic nevi. J Invest Dermatol 2014;134:1067-1074.

7 Ross AL, Sanchez MI, Grichnik JM: Molecular nevogenesis. Dermatol Res Pract 2011; 2011:463184.

8 Lu R, Li Q, Quan Y, Li K, Liu J: Staged surgery with total excision and lamellar reconstructive for medium-sized divided nevus of the eyelids. Plast Reconstr Surg Glob Open 2015; 3:e438.

9 Ghosh YK, Abu-Ain M, Ahluwalia HS: One stage management of a giant kissing naevus. Orbit 2010;29:174-175.
10 Morax S, Dhermy P, Sangare S: Giant pigmented nevus of the eyelids. J Fr Ophtalmol 1982;5:135-136.

11 Zaal LH, Mooi WJ, Klip H, van der Horst CMAM: Risk of malignant transformation of congenital melanocytic nevi: a retrospective nationwide study from the Netherlands. Plast Reconstr Surg 2005;116:1902-1909.

12 Fuchs A: Divided nevi of the eyelids. Urol Cutaneous Rev 1950;54:88-90.

13 Kharel Sitaula R, Batta S, Shrestha GB, Shrestha JK: Malignant transformation of kissing nevus - a rare entity. Nepal J Ophthalmol 2012;4:329-332.

14 Arad E, Zuker RM: The shifting paradigm in the management of giant congenital melanocytic nevi: review and clinical applications. Plast Reconstr Surg 2014;133:367-376. 